\title{
Small-scale topology of solar atmospheric dynamics
}

\section{Acoustic events and internetwork grains}

\author{
N. M. Hoekzema ${ }^{1}$, T. R. Rimmele ${ }^{2}$, and R. J. Rutten ${ }^{3}$ \\ 1 Max Planck Institut für Aeronomie, Max Planck Strasse 2, 37191 Katlenburg-Lindau, Germany \\ e-mail: hoekzema@linmpi.mpg.de \\ 2 National Solar Observatory/Sacramento Peak, PO Box 62, Sunspot, NM 88349-0062, USA \\ e-mail: rimmele@sunspot.noao.edu \\ 3 Sterrekundig Instituut, Postbus 80 000, 3508 TA Utrecht, The Netherlands \\ e-mail: R.J.Rutten@astro.uu.nl
}

Received 22 January 2002 / Accepted 22 April 2002

\begin{abstract}
We use high-quality observations from the Dunn Solar Telescope at NSO/Sacramento Peak to study spatio-temporal co-location of acoustic flux events in the photosphere and internetwork grains in the chromosphere. The events are diagnosed as sites with excess upward-propagating five-minute waves measured from Dopplergrams. The grains are repetitive bright internetwork features in simultaneous $\mathrm{Ca}_{\text {II }} \mathrm{K}_{2 \mathrm{~V}}$ filtergrams. We find that the largest-flux sites in the granulation have appreciably larger than random probability to co-locate with exceptionally bright chromospheric internetwork grains, at an average delay of about two minutes which is likely to represent sound travel time to the chromosphere. This finding strengthens the case for acoustic grain excitation.
\end{abstract}

Key words. Sun: photosphere - Sun: chromosphere - Sun: oscillations

\section{Introduction}

This paper combines two solar research topics that have received increasing attention over the past years: the excitation of sound waves by localized, small-scale "acoustic events" in the solar granulation, and the intermittent occurrence of "internetwork grains" in the low chromosphere. We use observations in which both phenomena were sampled simultaneously to address the question whether the one has something to do with the other. We find that they sometimes do when excessive in amplitude.

Acoustic events. The search for localized emission of acoustic waves in the solar photosphere started with the analysis of Goode et al. (1992) which suggested that the sources of the global solar $p$-mode oscillations may lie just below or even at the visible surface, even higher up than the shallow depths advocated by Kumar (1994, cf. Kumar \& Basu 1999). The search technique expanded the wave amplitude and phase diagnosis developed by Stebbins \& Goode (1987) in the context of gravity-wave detection (Stebbins et al. 1983), was refined by Restaino et al. (1993), and has been applied since by Rimmele et al. (1995), Goode et al. (1998) and Strous et al. (2000) in a sequence of "acoustic event" papers which established these as sources of enhanced acoustic wave generation which coincide spatially with intergranular lanes.

Send offprint requests to: R. J. Rutten,

e-mail: R.J.Rutten@astro.uu.nl
The events occur preferentially in intergranular lanes after small granules vanish abruptly, in excellent agreement with granular collapse predictions from theoretical studies and numerical simulations by Rast and Skartlien (Skartlien 1998; Rast 1999; Skartlien et al. 2000).

The identification of localized pistons for the five-minute oscillation bears also on issues in global helioseismology. The observed $p$-mode peak asymmetries and $V-I$ phase differences between $(k, \omega)$ ridges and interridges point to a correlation between the eigenmode oscillations and the diffuse modulation background (e.g., Nigam \& Kosovichev 1999a,b; Straus et al. 1999; Oliviero et al. 1999; Georgobiani et al. 2000; Skartlien $\&$ Rast 2000). Such a correlation may be attributed to acoustic events if these are appreciable contributors in both $p$-mode pistoning and local wave emission.

Internetwork grains. The chromospheric quiet-sun brightness features discussed here are known as $\mathrm{K}_{2 \mathrm{~V}}$ bright points, intranetwork points, and cell grains. We prefer to call them "internetwork grains" since they are not points but have spatial extent, are not part of the network ${ }^{1}$, and are present in

\footnotetext{
1 The network appears much more stable than the internetwork, see e.g., the illustrative TRACE movies at http://www.astro.uu.nl/ rutten/trace1. Note that the chromospheric network also consists of grains, each of which corresponds usually to a cluster of " $G$ band bright points" in the underlying photosphere (e.g., Lites et al. 1999). The present paper concerns internetwork grains only.
} 
ultraviolet images just as in $\mathrm{Ca}_{\mathrm{II}} \mathrm{K}_{2 \mathrm{~V}}$ or $\mathrm{H}_{2 \mathrm{~V}}$ filtergrams or spectroheliograms (Rutten et al. 1999; Krijger et al. 2001). They appear as intermittent brightening with three-minute repetitivity, superimposed on a slower-evolving mesh-shaped brightness pattern of which they represent local enhancements (Cram \& Damé 1983), and they often show fast apparent motion along the mesh strands (Lites et al. 1999).

A lengthy review of the extensive literature on this subject, dating back to Hale \& Ellerman (1904), led Rutten \& Uitenbroek (1991) to conclude that the internetwork grains are primarily acoustic in nature. The detailed reproduction of the complex $\mathrm{Ca}$ II $\mathrm{H}_{2 \mathrm{~V}}$ spectral behavior in the observations of Lites et al. (1993) that was achieved in the numerical radiationhydrodynamics simulation of Carlsson \& Stein (1997) confirmed beyond doubt that acoustic shock overtaking explains the spectral grain behavior.

A comprehensive analysis of seeing-free ultraviolet image sequences from the Transition Region and Coronal Explorer (TRACE) by Krijger et al. (2001) ties chromospheric Ca II $\mathrm{H} \& \mathrm{~K}$ and upper-photosphere dynamics together and demonstrates that acoustic oscillations are the primary ingredient of the "below-the-canopy" internetwork atmosphere. It also identifies the background mesh pattern as due to atmospheric gravity waves, and concludes that acoustic and gravity waves together constitute most of the dynamical behavior of the internetwork regions in the upper photosphere and low chromosphere.

Grain pistoning. What these studies do not clarify is the nature of the pistons that excite the waves making up internetwork grains. Just as for the five-minute oscillation, the question arises whether such excitation occurs all over the place or whether there exist localized sources of enhanced emission. The acoustic events generating five-minute power are broadband emitters and should also produce three-minute power. Turbulent convection causing the solar granulation is likely to produce atmospheric gravity waves in the overshoot regime. Internetwork magnetism, in a weaker form than the kilogauss fluxtubes making up the network, may play its own role in grain excitation and localization and in wave funneling and damping.

Carlsson \& Stein (1997) pointed out that their simulation produces realistic grains without magnetism, and numerous observers have sought for but not found an internetwork grainmagnetism connection (von Uexküll \& Kneer 1995; Steffens et al. 1996; Hofmann et al. 1996; Remling et al. 1996; Wellstein et al. 1998; Nindos \& Zirin 1998; Lites et al. 1999; Worden et al. 1999) other than the occasional "flashers" which seem to mark isolated fluxtubes on their way to or from the network (Brandt et al. 1992, 1994; Nindos \& Zirin 1998; Krijger et al. 2001). The attention has therefore turned to convective sound excitation and the question whether chromospheric grain occurrence may correspond to specific locations in the solar granulation.

The previous papers in this series (Hoekzema et al. 1998a,b; Hoekzema \& Rutten 1998; Hoekzema \& Brandt 2000) addressed this question through a statistical approach that we follow here as well. The conclusion from these analyses is that three-minute wave amplitudes and $\mathrm{K}_{2 \mathrm{~V}}$ grain occurrence peak above intergranular lanes, especially in mesoscale convergence areas. It implies that analyses addressing pistoning of chromospheric three-minute oscillations and analyses addressing excitation of the global five-minute oscillation both regard specific intergranular events as sources of enhanced wave emission.

Nevertheless, the suspicion that internetwork magnetism plays a dominant role in internetwork grain localization has not been laid to rest. It started with the often-disputed claim of a one-to-one correlation, even with direct amplitude proportionality, between the presence of patches of enhanced internetwork field and the presence of $\mathrm{K}_{2 \mathrm{~V}}$ grains by Sivaraman \& Livingston (1982), later supported by Damé and coworkers (Damé et al. 1984; Damé 1985; Damé \& Martić 1987, 1988) and repeated by Sivaraman (1991). A recent combined effort (Sivaraman et al. 2000) restates the case for such a direct magnetic connection by again claiming high correlation between internetwork field enhancements in magnetograms and grain presence in $\mathrm{K}_{2 \mathrm{~V}}$ spectroheliograms.

Event-grain correspondence search. In this paper we combine the acoustic-event searching technique initiated by Restaino et al. (1993) with the statistical "correspondence" evaluation employed in the earlier papers of this series to search for a relationship between acoustic events in the photosphere and internetwork grain occurrence in the chromosphere. We find appreciably larger than random coincidence between the two when their amplitudes are large, and so add fuel to the grain pistoning debate.

\section{Observations and reduction}

Observations. We use multi-wavelength image sequences taken at the Dunn Solar Telescope of the US National Solar Observatory at Sacramento Peak in New Mexico on September 27, 1998.

The Universal Birefringent Filter (UBF) was used to scan the profile of the magnetically insensitive (Lande factor $g=$ 0) Fe I $5434 \AA$ line. Although the UBF passband (140 m $\AA$ ) was significantly wider than for the UBF plus Fabry-Perot filter combination employed in the earlier acoustic event observations, the spectral resolution remains sufficient to measure Dopplershifts at different photospheric heights by measuring the line wing positions at different intensity levels. The UBF filtergrams were recorded with a $1 \mathrm{~K} \times 1 \mathrm{~K}$ CCD camera. In addition, $\mathrm{Ca}$ II $\mathrm{K}_{2 \mathrm{~V}}$ filtergrams were recorded using a second CCD camera placed behind a Halle tunable Ca II $\mathrm{K}$ filter. Its passband was set to $F W H M=0.6 \AA$ centered on the $\mathrm{Ca}_{\mathrm{II}} \mathrm{K}_{2 \mathrm{~V}}$ emission feature. $\mathrm{K}_{2 \mathrm{~V}}$ exposures were taken at the start and completion of each UBF scan and averaged.

The field of view was approximately $165 \times 165 \mathrm{arcsec}^{2}$ for both the UBF and the $\mathrm{Ca}$ II $\mathrm{K}_{2 \mathrm{~V}}$ filtergrams. The telescope was pointed to a quiet area at the exact center of the apparent solar disk, using a correlation tracker to stabilize the image. The pixel resolution was $0.17 \mathrm{arcsec} /$ pixel for the UBF and 0.33 arcsec/pixel for the $\mathrm{Ca}_{\mathrm{II}} \mathrm{K}_{2 \mathrm{~V}}$ filtergrams. The exposure 


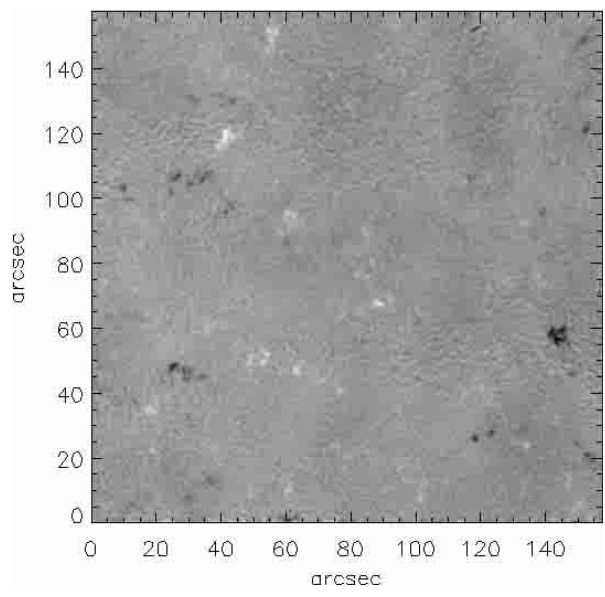

Fig. 1. Time-averaged magnetogram determined from $\mathrm{Fe}_{\mathrm{I}} 6302 \AA$ A. The flux density range is approximately \pm 15 Gauss. The observed disk-center area was very quiet. The black and white patches mark clusters of kilogauss magnetic elements, of opposite polarity respectively, which together constitute sparse bipolar network. The internetwork covering the remainder of the field shows mainly noise. Axis scales: 1 arcsec corresponds to $0.72 \mathrm{Mm}$ on the sun.

times were about $200 \mathrm{~ms}$ for the UBF filtergrams and $2 \mathrm{~s}$ for the $\mathrm{Ca}$ II $\mathrm{K}_{2 \mathrm{~V}}$ images.

The profile of the Fe I $5434 \AA$ line was scanned repetitively by stepping the UBF transmission profile through it in $20 \mathrm{~m} \AA$ steps. Each profile scan covered 14 wavelength positions including the continuum. In addition, a Fe I $6302 \AA$ magnetogram was recorded at the beginning of each scan using the UBF in magnetograph mode, switching a quarter-wave plate to obtain images in lefthand and righthand circular polarization. The line-of-sight magnetograms that result from their subtraction are not used in this analysis (their sensitivity is insufficient to identify internetwork fields) but a sample is shown in Fig. 1 to demonstrate the quietness of the field of view. It took about $40 \mathrm{~s}$ to complete one full UBF wavelength-stepping sequence. An observing sequence spanning a full hour was obtained under good to excellent seeing conditions.

Initial reduction. The data reduction included dark current and flat field corrections and removal of atmospheric distortion of the filtergrams using a destretching algorithm. The UBF filtergrams and the $\mathrm{Ca}_{\text {II }} \mathrm{K}_{2 \mathrm{~V}}$ filtergrams were precisely co-aligned using simultaneous images of an standard target raster placed at the prime focus of the telescope.

Large-scale transmission gradients of instrumental origin were removed from the UBF images through scaling to a smoothed local-area average. Three-dimensional "subsonic" $(k, \omega)$ filtering following Rimmele et al. (1995) was used for the granulation data to remove the interference patterns of the fiveminute oscillation and other modulations with apparent horizontal motions in excess of the sound speed. Reverse filtering was applied to the flux maps to isolate the five-minute modulation signal. (The global $p$-modes are removed from these through their vertical phase signature in the flux definition described below.)
The edges of the field of view were removed because they are affected by the apodization required for Fourier filtering. The Ca II $\mathrm{K}_{2 \mathrm{~V}}$ images do not need gradient subtraction, were not Fourier-filtered, and were cut in size to the field of view of the granulation images and flux maps.

The final product of the reduction consists of three co-aligned and synchronous "image cubes", each containing 78 images of $158 \times 158 \operatorname{arcsec}^{2}$ at 47 s cadence, respectively of the photospheric intensity (granulation), the chromospheric Ca II $\mathrm{K}_{2 \mathrm{~V}}$ intensity, and the acoustic flux defined below.

Figure 2 shows sample images. They illustrate the large size of the field of view used in our analysis, an important asset through providing good statistics.

The insets in Fig. 2 show the result of the histogram equalization that we apply to all data when measuring "correspondence" as described below. Such amplitude redistribution enhances the relative importance of the locations making up the tails of the brightness distribution. In particular, it enhances the internetwork grains in the $\mathrm{Ca}$ II $\mathrm{K}_{2 \mathrm{~V}}$ filtergrams.

Acoustic flux measurement. The derivation of acoustic flux from the Fe I $5434 \AA$ filtergrams taken with the UBF followed the procedure of Restaino et al. (1993) and Rimmele et al. (1995). For each spatial pixel and each wavelength scan through the line profile, Dopplershifts were measured at different intensity levels in the line profile, representing different formation heights $z$ in the photosphere. The resulting $v(x, y, t)$ Dopplershift cubes were $(k, \omega)$-filtered to select the five-minute oscillation band. Hilbert transformation then delivers the velocity amplitude and phase as function of space and time. Finally, acoustic flux is defined by

$$
u(x, y, t) \equiv c \frac{v_{5}^{2}}{\omega} \frac{\Delta \phi}{\Delta z},
$$

where $c$ is a constant, $v_{5}$ the radial velocity in the five-minute band, $\omega$ the frequency, and $\Delta \phi / \Delta z$ the vertical phase gradient taken positive for upward propagating waves. In this analysis we use the deepest part of the line to obtain a measure of $u(x, y, t)$ in the middle photosphere. Note that the solar $p$-mode eigen-oscillations in the five-minute band consist of evanescent waves in the photosphere and vanish from $u$ through having $\Delta \phi / \Delta z=0$.

The third image in Fig. 2 shows the resulting acoustic flux map for the same moment for which the other two display photospheric and chromospheric brightness. The flux map displays a finely textured distribution over the surface with many locations where large positive (white) and large negative (dark) flux appear together in close-lying patches, causing bipolar appearance. It is not clear whether the relatively smooth intervening grey areas contain intrinsically smaller flux or whether they contain similar but yet smaller bipolar combinations that remain unresolved in our data and therefore cancel through resolution smearing.

Time slices. Figure 3 adds a sample of temporal behavior to the spatial samples of our data in Figs. 1-2 by displaying "time slice" cuts through the three data cubes. They sample 

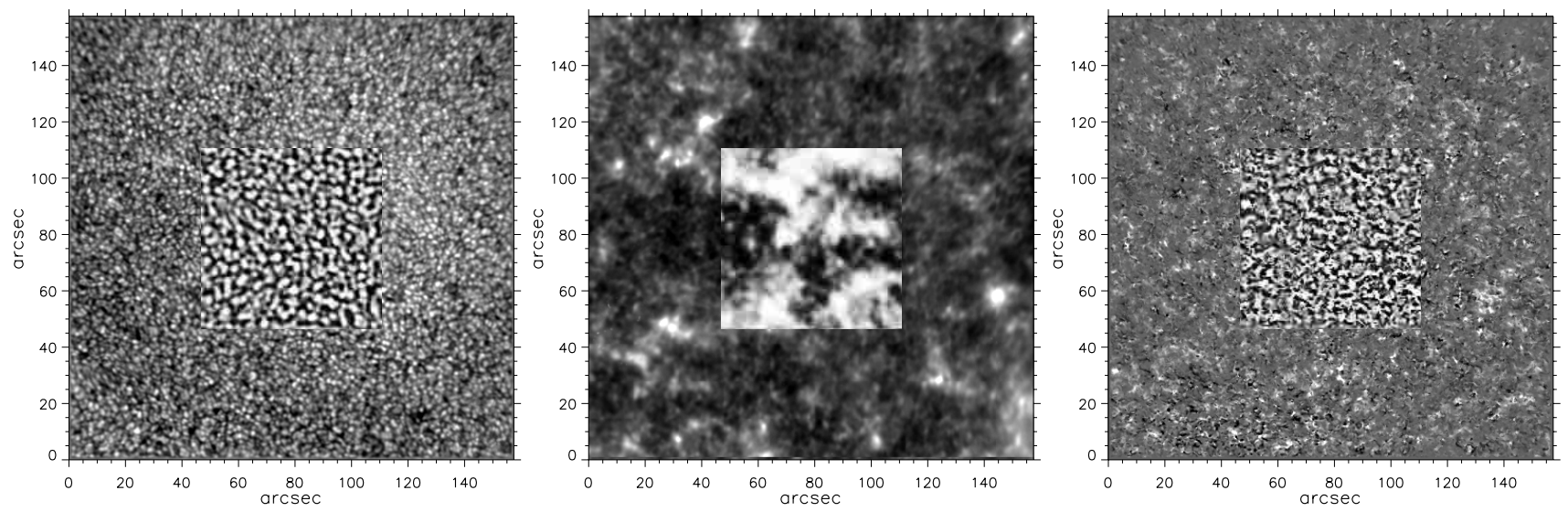

Fig. 2. Sample images (No. 10 of each data cube). Left: granulation after gradient removal. The inset shows the magnified lower-left corner (45x 45 arcsec) after subsonic filtering and histogram equalization. Middle: co-temporal $\mathrm{Ca}$ II $\mathrm{K}_{2 \mathrm{~V}}$ image. The brightest parts mark chromospheric network and are co-spatial with the magnetic network in Fig. 1. Inset: magnified lower-left corner after histogram equalization. Internetwork grains are less bright than the network and gain visibility in the inset (small bright features in dark areas). The bright network areas are excluded from the analysis. Right: co-temporal acoustic flux map, showing the surface distribution of the propagative five-minute wave flux measured from Fe I 5434 Å. Bright: upward propagation. Dark: downward propagation. Inset: magnified lower-left corner after histogram equalization.
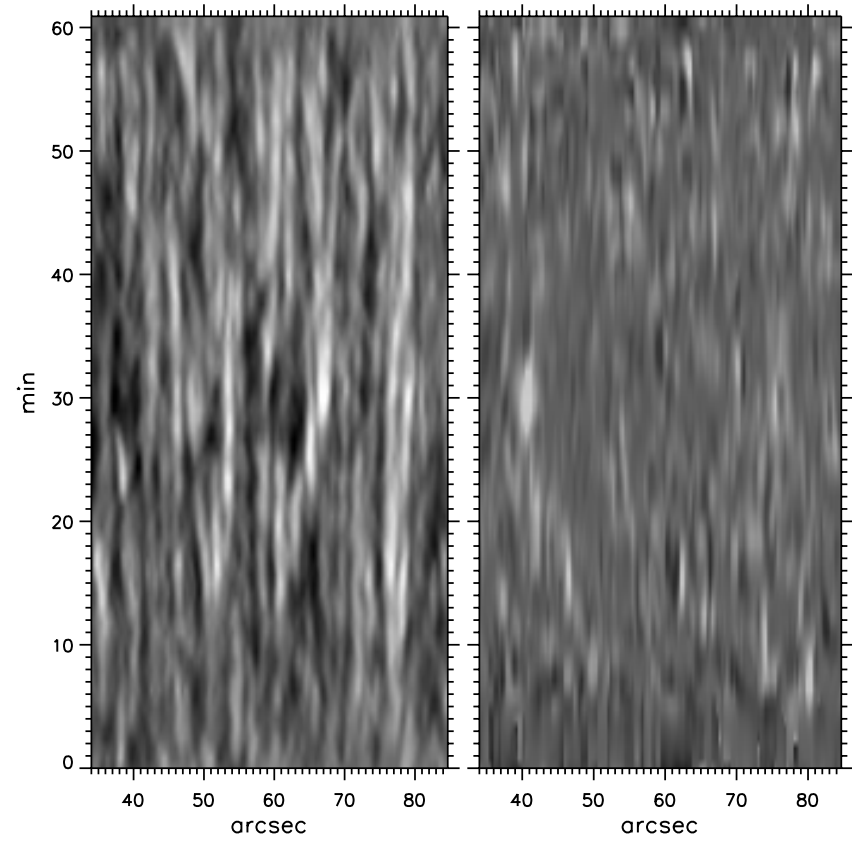

the evolution of the measured quantity with time for a segment along one spatial axis, in this case $x=34-85 \operatorname{arcsec}$ at $y=76.5$ arcsec. A short segment is chosen to obtain sufficient magnification in the figure. Its selection was more or less random, simply choosing an internetwork area bordered by network, but even this small sample is characteristic for granular brightness, acoustic flux and $\mathrm{Ca}$ II $\mathrm{K}_{2 \mathrm{~V}}$ brightness behavior.

The $\mathrm{K}_{2 \mathrm{~V}}$ slice at right shows that there is network in this segment around $x=81 \mathrm{arcsec}$. It produces bright vertical striping which is overexposed because the grey scale was clipped to half the full range for internetwork enhancement (cf. $y$-axis length difference of Figs. 5 and 6 below). There is also a narrow stripe of extended brightness at $x=45$ arcsec, located in the internetwork but longer-lived than the oscillation patterns which give the internetwork its characteristic dappled

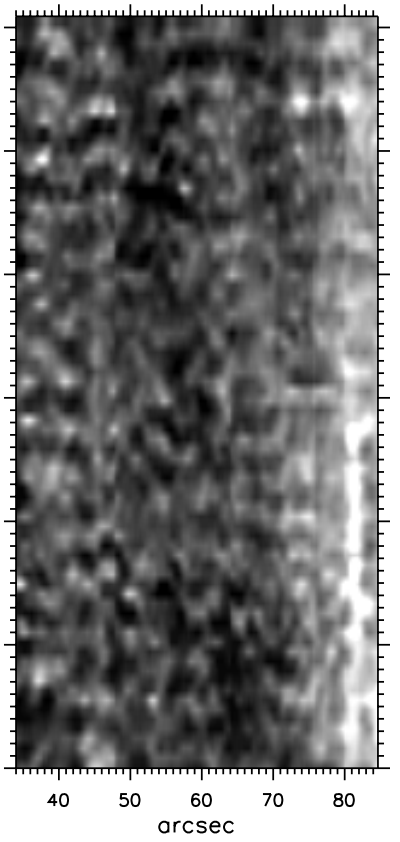

Fig. 3. Spatio-temporal "time slices" for a partial cut through the three data cubes, at $y=76.5$ arcsec along the $x$-segment specified horizontally. Left: granular brightness evolution. Middle: acoustic flux. Right: Ca II $\mathrm{K}_{2 \mathrm{~V}}$ brightness, with the greyscale clipped to half the observed range.

time-slice appearance. We suspect this to be a magnetically anchored internetwork flasher (cf. Krijger et al. 2001) which betrays its presence here by not moving "off the slit" in the $y$-direction.

The $\mathrm{K}_{2 \mathrm{~V}}$ internetwork oscillation patterns are mostly weak and fuzzy with "wavy curtain" interference patterns. The local brightenings, which constitute the internetwork grains seen in $\mathrm{K}_{2 \mathrm{~V}}$ movies, occasionally become rather bright, as in the strip at left with $x<40$ arcsec. They would sharpen and brighten yet more at narrower $\mathrm{K}_{2 \mathrm{~V}}$ bandwidth than the $0.6 \AA F W H M$ used here, but the spatio-temporal pattern remains similar at wider bandwidth (see passband comparisons in Fig. 9 of Krijger et al. 2001).

The slice at left in Fig. 3 shows characteristic granular evolution patterns. The network area at the far right contains no 


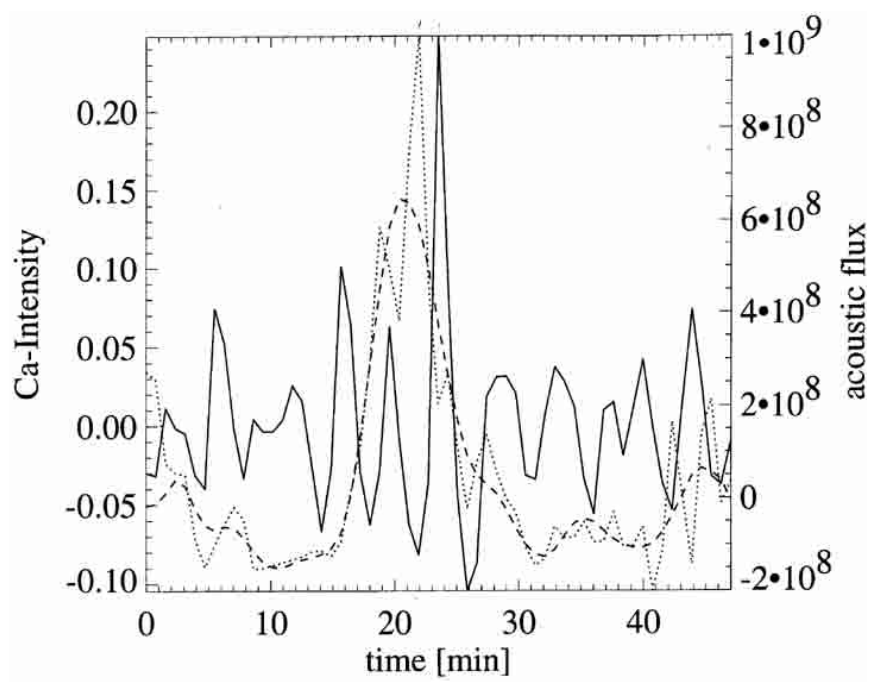

Fig. 4. Internetwork acoustic flux event with concomitant Ca II $\mathrm{K}_{2 \mathrm{~V}}$ intensity. The solid curve (scale at left, fractional deviation from the mean value with the mean subtracted) shows the temporal behavior of $\mathrm{K}_{2 \mathrm{~V}}$ brightness at a selected internetwork location. The dashed curve (scale at right, in erg cm $\mathrm{cm}^{-2} \mathrm{~s}^{-1}$ but rather imprecise) is the acoustic flux in the five-minute band as defined by Eq. (1) and used in this paper. The dotted curve shows the acoustic flux determined with the inclusion of frequencies above the acoustic cutoff $f \approx 5.5 \mathrm{mHz}$.

bright granule during the hour of observation, but bright granules crop up repeatedly just to the left of it.

The acoustic flux slice in the middle of Fig. 3 contains one exceptionally bright event at $x \approx 41 \operatorname{arcsec}, y \approx 30 \mathrm{~s}$. The $\mathrm{K}_{2 \mathrm{~V}}$ slice shows a well-defined grain train around that spatiotemporal location. Its grains are not spectacularly bright but its oscillatory behavior, with deep dark minima between the grains, is relatively pronounced.

Eyeball comparison of these three slices (a better option is to play such data cubes as movies or to cut three-dimensionally through them with cursor control on a computer screen) shows that there is much dynamical change in the photosphere and chromosphere without obvious one-to-one relationships between the different phenomena.

\section{Analysis and results}

Example event. We first give an indicative example (which actually triggered this analysis) in Fig. 4. It shows the temporal behavior of acoustic flux and $\mathrm{Ca}$ II $\mathrm{K}_{2 \mathrm{~V}}$ brightness at a selected internetwork pixel at which an internetwork grain occurred (sharp peak in the solid curve at $t=23.5 \mathrm{~min}$ ) a few minutes after a flux event (broad peak around $t=21 \mathrm{~min}$ in the dashed curve). The graph obviously suggests that the event caused the grain, with the delay of a few minutes corresponding to the sound travel time from photosphere to chromosphere.

The dotted curve results when the acoustic flux is evaluated including higher-frequency components than the five-minute band. The difference between the two flux determinations in the peak region indicates that higher-frequency waves may play a role in internetwork grain generation. They are not accounted for in the flux determinations in this paper, but should be included in future analyses of observations with higher time resolution than given by our $47 \mathrm{~s}$ image cadence.

Scatter diagram. We now turn to statistical methods to answer the question whether the example in Fig. 4 is a "typical" one that characterizes general behavior or just a misleading freak coincidence between peaks in flux and $\mathrm{Ca}$ II $\mathrm{K}_{2 \mathrm{~V}}$ brightness. The naïve way to search for spatial correlation between two such quantities is to simply plot the amplitude of the one against the amplitude of the other per pixel in two simultaneous images. Such a scatter plot between acoustic flux and $\mathrm{K}_{2 \mathrm{~V}}$ brightness is shown in Fig. 5 but in a rather elaborate format developed by Strous (1994). The format is used in Fig. 6 to search for time-delay correlation; Fig. 5 serves to introduce it and the procedure.

Both the acoustic flux and the $\mathrm{K}_{2 \mathrm{~V}}$ brightness images were spatially smoothed through $2 \times 2$ pixel boxcar averaging to reduce noise and remaining co-alignment errors. Ten successive image pairs were used to generate 2 million pixel-by-pixel comparisons. These are plotted in Fig. 5, using point density contours rather than the samples themselves except for the outermost part of the scatter cloud. The density distributions, plotted invertedly along the top and right-hand sides, show that the outer contour samples very low point density and that the central mountain would turn solidly black if the higher-density areas were plotted as individual sample points.

Both distributions are skewed, possessing extended tails toward high values. The center of gravity lies asymmetrically at the lower left. For the acoustic flux this asymmetry shows that large upward flux occurs more often than equally large downward flux, producing contour extensions to the right. For the $\mathrm{K}_{2 \mathrm{~V}}$ brightness the skewing results from having both network and internetwork present in the field of view, with the bright network producing upward contour extensions. Both extensions are particularly large for the outer contour, corresponding to the very far tails of the distributions (largest amplitudes occurring only rarely).

The dashed curves are the first moments of the distributions for one quantity per bin in the other quantity. If $\mathrm{K}_{2 \mathrm{~V}}$ brightness would vary in direct proportion to acoustic flux at each pixel, the scatter points would cluster along the forward diagonal and both moment curves would also bend toward the forward diagonal. Clustering and bending toward the backward diagonal would mean anti-correlation. The perpendicularity of the moment curves in Fig. 5 indicates absence of correlation.

Time-delay scatter diagrams. Figure 6 results from similar scatter measurements but after adding two refinements. Firstly, the network has been taken out of the pixel-by-pixel sampling by making a mask that deletes pixels with large temporally-averaged $\mathrm{K}_{2 \mathrm{~V}}$ brightness. It is set conservatively and transmits only a quarter of the total area. Secondly, the two diagnostics are now measured adding image number offsets $\Delta N=[-19,-4,-2,-1,0,1,2,3,4,5,10,19]$ between the two ten-image sequences. Since the imaging cadence was $47 \mathrm{~s}$, these offsets produce the time delays $\Delta t$ specified in each panel. 


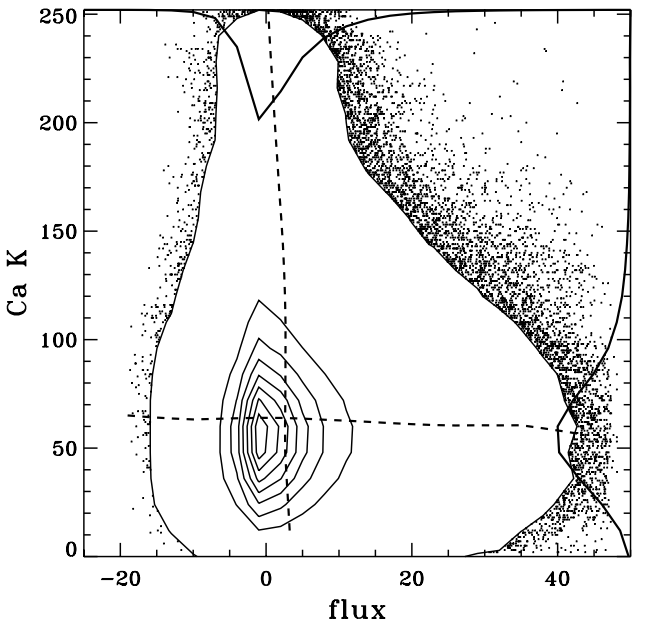

Fig. 5. Strous-format scatter density plot, showing pixel-by-pixel scatter of $\mathrm{Ca}$ II $\mathrm{K}_{2 \mathrm{~V}}$ brightness against acoustic flux measured for the whole field in 10 image pairs. Axis scales: arbitrary units. Point density contours are plotted instead of single points at large point density to avoid overcrowding, with equal point density increments per contour. The solid curves along the top and righthand axes show the occurrence distributions on inverted and normalized scales. Both have extended large-value tails. The dashed curves are the first moments of the point density distribution per bin in each quantity. Their perpendicularity indicates absence of correlation between flux amplitude and simultaneous $\mathrm{K}_{2 \mathrm{~V}}$ brightness, but such correlation emerges in Fig. 6.

The values were selected to cover half an hour with good sampling over $\Delta t=-1.5$ to $+4 \mathrm{~min}$.

The $\Delta t=0 \mathrm{~s}$ panel duplicates Fig. 5 but only for the internetwork pixels. The $\mathrm{K}_{2 \mathrm{~V}}$ brightness scale reaches only half as high because the brighter half exclusively came from network. Deleting those pixels delivers a small but significant rightward bend in the vertical moment curve at $\Delta t=0$, reaching maximal offset where it crosses the outer contour. Taking the latter as significance delimiter, the rightward bend implies significant correlation between exceptionally large internetwork flux and maximum internetwork $\mathrm{K}_{2 \mathrm{~V}}$ brightness. The similar rightward curving of the vertical moment curve below the mountain summit indicates similar correlation between large flux and minimum internetwork $\mathrm{K}_{2 \mathrm{~V}}$ brightness.

These correlations are very far from one-to-one (= purely diagonal scatter clouds and moment curves) but they are nevertheless highly significant since the sampling statistics are excellent ( 0.5 million pixels remain after the network maskoff). They indicate that, amidst other processes governing $\mathrm{Ca}$ II $\mathrm{K}_{2 \mathrm{~V}}$ brightness, the local acoustic flux is an additional $\mathrm{K}_{2 \mathrm{~V}}$ brightness-contributing agent of which the share increases where it is exceptionally large.

The other panels of Fig. 6 show similar density contour plots for non-zero time delays, both negative $\left(\mathrm{K}_{2 \mathrm{~V}}\right.$ sampled before flux) and positive $\left(\mathrm{K}_{2 \mathrm{~V}}\right.$ after flux). The first panel repeats the full Strous format of Fig. 5 in order to show the $\mathrm{K}_{2 \mathrm{~V}}$ brightness distribution curve for internetwork only: it is nearly symmetric. This panel is for $15 \mathrm{~min}$ time delay, as is the last panel for the opposite sign; these extreme panels display near-perfect perpendicularity of the moment curves, signifying that at such
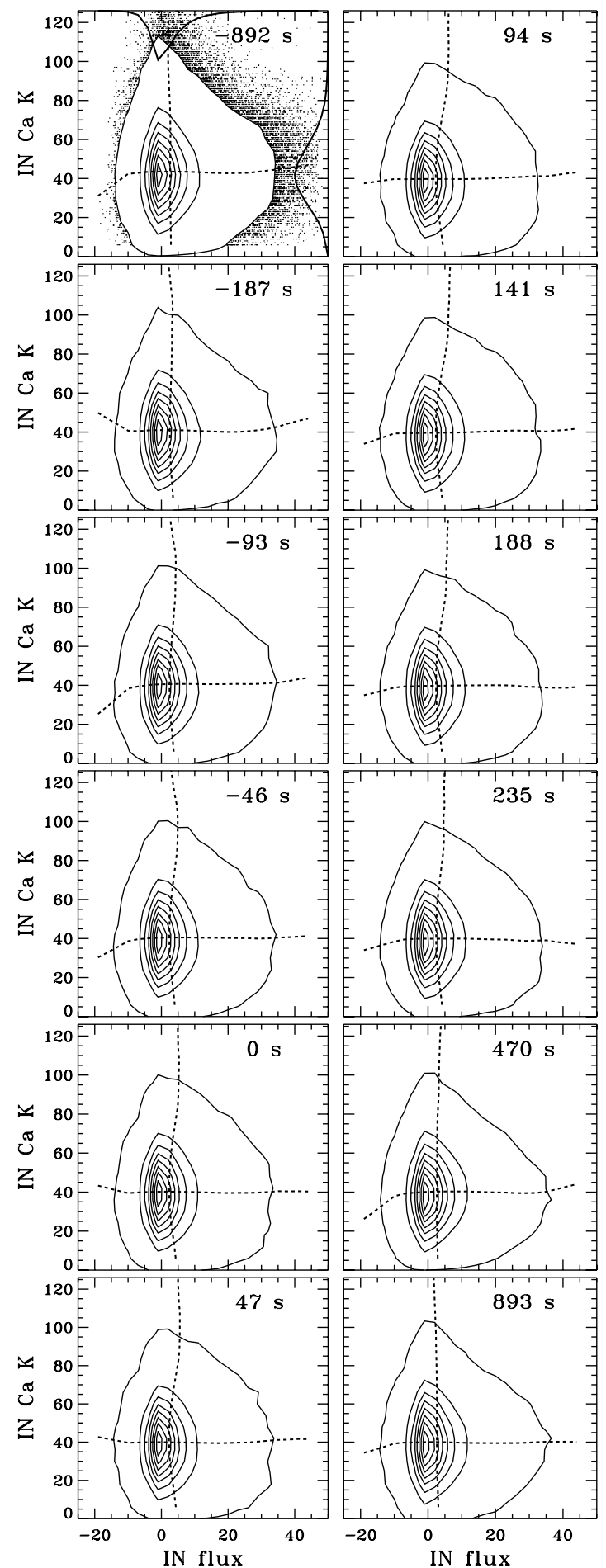

Fig. 6. Time-delay scatter density plots for acoustic flux and $\mathrm{Ca}$ II $\mathrm{K}_{2 \mathrm{~V}}$ brightness, internetwork (IN) area only. Each panel compares the two quantities per internetwork pixel from 10 successive image pairs at different time delay $\Delta t$ (specified per panel) between the two sequences, positive $\Delta t$ denoting $\mathrm{K}_{2 \mathrm{~V}}$ sampling after flux sampling. The first panel repeats the full Strous format shown in Fig. 5 at half the vertical axis range. In the other panels the individual sample points outside the outer contour and the distribution curves are left out to reduce plot file size and clutter (the distributions are the same for each panel anyhow). The rightward bending of the upper part of the vertical moment curve indicates small but significant correlation between extreme internetwork $\mathrm{K}_{2 \mathrm{~V}}$ brightness and large upward acoustic flux during $\Delta t \approx-1$ to $+5 \mathrm{~min}$. 
large time delay there is no correlation whatsoever between the two signals.

The intermediate sequence of time-delay panels in Fig. 6 clearly shows the presence of significant (but not exclusive) correlation for time delays ranging from $\Delta t \approx-1$ to $+5 \mathrm{~min}$, with a gradual increase until $\Delta t \approx+2 \mathrm{~min}$. The conclusion is that excessive flux is likely to be accompanied by excessive internetwork $\mathrm{K}_{2 \mathrm{~V}}$ brightness at an average delay of a few minutes. This does not happen in one-to-one causality, but occurs frequently enough to emerge as a systematic trend.

Correspondence. The above results show that acoustic flux and $\mathrm{Ca}$ II $\mathrm{K}_{2 \mathrm{~V}}$ brightness go together when excessively large at least some of the time. The partial nature of this apparent correlation suggests the use of methods that isolate such specific behavior in the presence of other processes not partaking in the correlation. We therefore turn to using the "correspondence" $C$ introduced by Hoekzema et al. (1998b) to evaluate partial spatio-temporal coincidence rates. This statistical measurement of coincidence likelihood can be evaluated reliably from large data sets for very selective pixel classes with small filling factor, in the presence of multiple patterning agents which confuse straightforward correlation. In this case we employ it to quantify the co-occurrence likelihood between acoustic flux events and $\mathrm{K}_{2 \mathrm{~V}}$ grains and the temporal lag between such co-occurrences.

The correspondence $C$ measures selective co-location likelihood defined by

$C \equiv \frac{f_{\mathrm{AB}}}{f_{\mathrm{B}}}=\frac{N_{\mathrm{AB}} / N_{\mathrm{A}}}{N_{\mathrm{B}} / N}$,

where the coincidence filling factor $f_{\mathrm{AB}}=N_{\mathrm{AB}} / N_{\mathrm{A}}$ specifies the fraction of pixels of type $A$ in the internetwork part of one image or map that also belong to type $\mathrm{B}$ in another image or map, and the filling factor $f_{\mathrm{B}}=N_{\mathrm{B}} / N$ measures the spatial occurrence frequency of the second category over the internetwork part of the field of view made up of $N$ pixels. The normalization of $f_{\mathrm{AB}}$ by $f_{\mathrm{B}}$ yields a probability scale where $C=1$ suggests that $\mathrm{A}$ and $\mathrm{B}$ are independent phenomena, values $C>1$ imply that pixels of type A are preferentially co-located with pixels of type $\mathrm{B}$, and values $C<1$ imply spatial avoidance. Thus, $C$ specifies the co-location probability in terms of the random-draw likelihood. Its value does not change when exchanging $\mathrm{A}$ and $\mathrm{B}$ and does not depend on the amplitude of each signal, only on its spatial distribution. The maximum, $C_{\max }=1 / f_{\mathrm{B}}$, is reached when all pixels of type A co-locate with pixels of type $B$.

We plot correspondence $C$ against time delay $\Delta t$ in Figs. $7-$ 10 below. When $\mathrm{A}$ and $\mathrm{B}$ describe pixels sharing the same property but sampled at different times, $C(\Delta t)$ measures selfcorrespondence as function of time, i.e., the persistency of the specific property as a decrease from $C(\Delta t=0)=1 / f_{\mathrm{B}}$. In general, $C(\Delta t)$ quantifies time-delay correspondence when $\mathrm{A}$ and $\mathrm{B}$ differ in nature and the maps that are compared were taken at a time interval $\Delta t$. The resulting $C$ peaks at a value $\Delta t$ when phenomenon A is most likely to be followed or preceded by phenomenon B at that time delay.

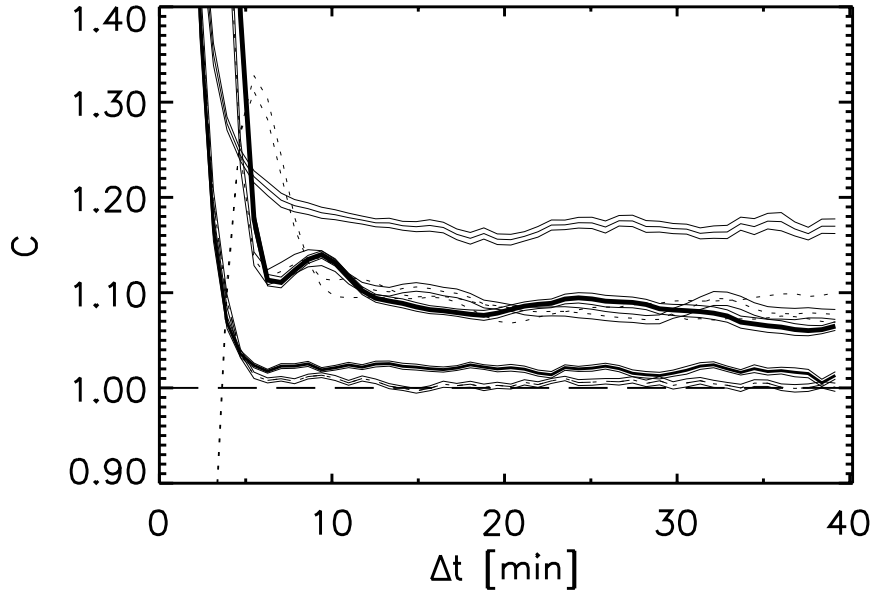

Fig. 7. Persistency and cross-correspondence $C$ of acoustic flux as a function of time delay $\Delta t$, averaged over all internetwork pixels. Each curve is bordered by $1 \sigma$ variance estimates. The solid curves show self-correspondence $C$ for different $10 \%$ decadal bins in histogramequalized flux $u$ as shown in the inset of Fig. 2, with zero flux at $50 \%$. The upper curve reaching $C \approx 1.17$ at right holds for the $45-54 \%$ minimum-flux bin. The pair of curves with $C \approx 1.08$ at right are for the maximum (90-99\%) upward and downward (0-09\%) flux bins. The two curves near $C=1$ at right are for intermediate flux values, respectively upward (70-79\% bin) and downward (20-29\% bin). The dotted curves peaking at $\Delta t=6 \mathrm{~min}$ describe the time-delay crosscorrespondence between the largest upward and downward flux (upafter-down and down-after-up, respectively).

The correspondence analysis starts with histogram equalization of both types of image or map. This serves to define histogram bins as different pixel categories with the same number of pixels, equalizing their statistical weights. We mostly choose the extreme $5 \%$ bins below in our correspondence evaluations, and so are very selective in pixel subset selection. Nevertheless, the results gain high significance from large sampling statistics because the large field of view and the 78-image sequence length provide large numbers of pixels to average over.

Time-dependent network masks were constructed for the correspondence analyses by first smoothing and then averaging nine consecutive $\mathrm{K}_{2 \mathrm{~V}}$ images bracketing the moment of map acquisition. The internetwork areas darken with respect to the network through this procedure because internetwork grains are small and occur intermittently. After such averaging a brightness cutoff (at $80 \%$ after histogram equalization) defines an appropriate mask. Only the pixels in the remaining internetwork area of any map were used to measure the correspondences shown below.

Flux versus flux. Figure 7 shows persistency and time-delay cross-correspondence for the acoustic flux in internetwork areas. The overall persistency picture is one of rapid decline from the $C=10$ maximum per decadal percentile. The longest lifetime is shown by the maximum-flux sites, but even these acoustic events last less than five minutes.

At longer delay the intermediate-flux sites show persistency equal to the random likelihood, but the extreme-percentile curves in the middle indicate that after an event of maximum 


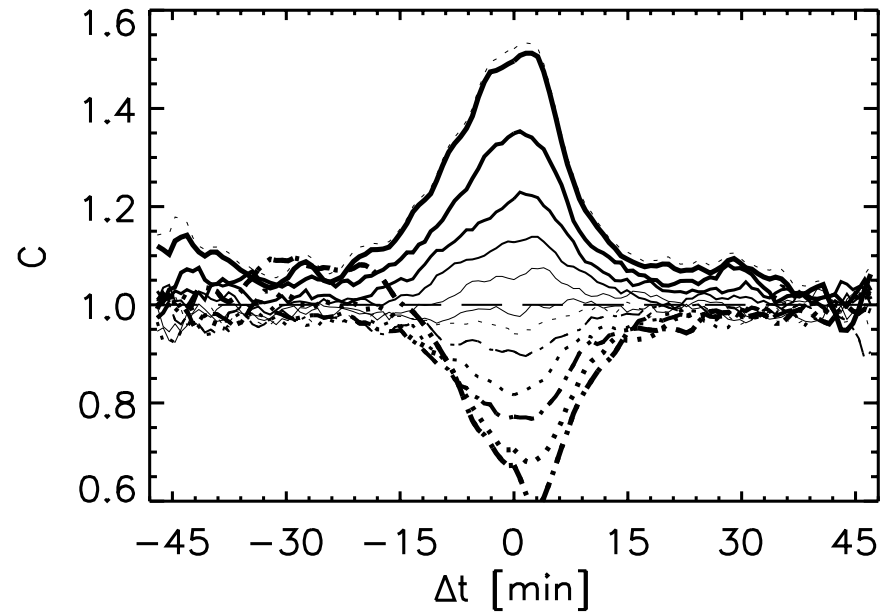

Fig. 8. Time-delay correspondence between excessive acoustic flux and granulation brightness for the 5\% bin of internetwork pixels with maximum upward propagating flux. The 12 curves specify their colocation probability with pixels in the following $5 \%$ bins of granular brightness: $0-5 \%$ (darkest intergranular lanes, top curve, with $1 \sigma$ variance), 5-10\%, 10-15\%, 20-25\%, 30-35\%, ..., 90-95\%, 95-100\% (brightest granules, lowest curve).

upward or downward flux, that site has a slightly but significantly enhanced probability of producing another maximumflux event during the 40-min delay measurement range. The curves for upward and downward flux are about the same. The zero-flux sites show yet larger statistical persistency, $17 \%$ probability over the random likelihood. An obvious speculation suggests that low acoustic flux and persistency go together at sites with enhanced internetwork magnetism, and that the presence of a few such locations sets this statistical preference.

The dotted pair of curves starting at $C=0$ measure the cross-correspondence between the decadal percentiles with the largest upward and downward flux. They peak at $C \approx 1.3$ for $\Delta t=5 \mathrm{~min}$, suggesting that extreme flux events of either sign have a small but significant probability to be followed by equally extreme flux events of the opposite sign. Downflow followed by upward wave generation is a feature of the wave excitation mechanism of Rast (1999) and Skartlien et al. (2000). The reverse correlation (down after up) has the same correspondence behavior. It may mark sites with large wave reflection.

Granulation versus flux. Figure 8 displays time-delay correspondence between pixels with excessive acoustic flux (the 5\% bin with maximum upward flux amplitude) and granular brightness, spread in $5 \%$ bins over the whole granulation brightness range. The probability that maximum upward flux is located at the very darkest intergranular lanes is $50 \%$ larger than the random likelihood. There is a smooth progression with increasing brightness to reverse "avoiding" of the brightest granules (lowest curve).

The curves in Fig. 8 are wide due to the granular evolution time scale. They peak at delay $\Delta t \approx 2 \mathrm{~min}$, implying that when the acoustic flux reaches excessive maxima, it tends to peak a few minutes after maximum intergranular darkness.

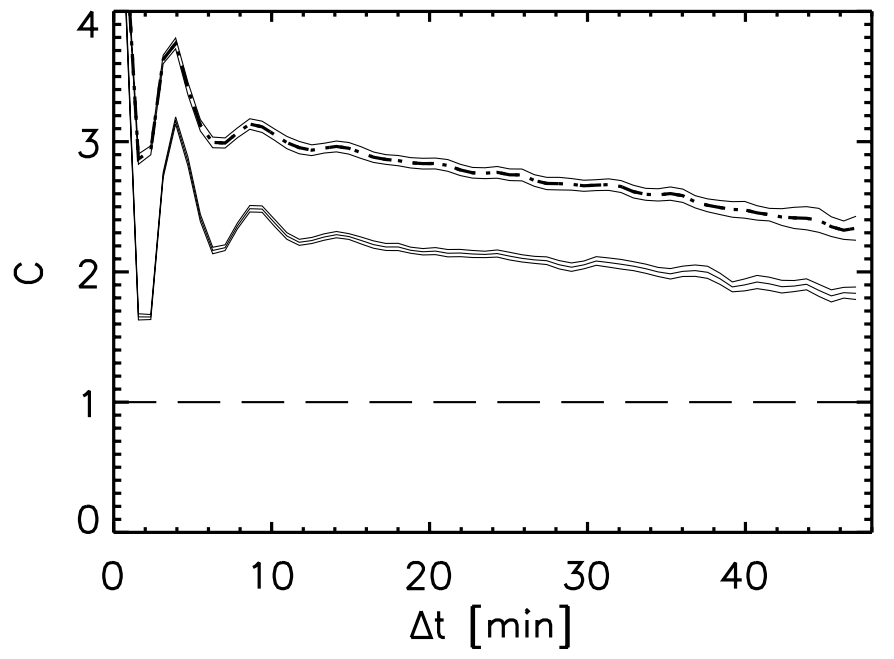

Fig. 9. Time-delay self-correspondence for extreme internetwork Ca II $\mathrm{K}_{2 \mathrm{~V}}$ brightness and darkness, with $1 \sigma$ variances. Upper curve: $10 \%$ bin of the pixels with the lowest internetwork $\mathrm{K}_{2 \mathrm{~V}}$ brightness. Lower curve: $10 \%$ bin with the largest internetwork $\mathrm{K}_{2 \mathrm{~V}}$ brightness.

This pattern that acoustic events preferentially occur at dark intergranular lanes and with the acoustic flux reaching its maximum value a few minutes after maximum intergranular darkness confirms the results of Rimmele et al. (1995) and Strous et al. (2000).

$\mathrm{K}_{2 \mathrm{~V}}$ versus $\mathrm{K}_{2 \mathrm{~V}}$. Figure 9 displays self-correspondence for the $10 \%$ pixel bins with the largest and the smallest internetwork Ca II $\mathrm{K}_{2 \mathrm{~V}}$ brightness, respectively. The very high correspondence peaks at $\Delta t=3-4 \mathrm{~min}$ and the diminishing peaks at multiple delay values illustrate the oscillatory behavior of internetwork $\mathrm{K}_{2 \mathrm{~V}}$ brightness, often showing a few cycles at such periodicity in time slices as in the righthand panel of Fig. 3.

The brightest $10 \%$ curve reaches lower correspondence than the darkest $10 \%$ curve, possibly due to the presence of occasional internetwork flashers that contribute longer-lived emission while migrating through the field of view (cf. Brandt et al. 1992, 1994; Krijger et al. 2001). The dark minima are a cleaner diagnostic of the oscillatory component of internetwork brightness behavior.

Flux versus $\mathrm{K}_{2 \mathrm{~V}}$. Our principal correspondence measurement concerns the internetwork co-location likelihood of flux events (which we now classify as the extreme 5\% pixel bins of upward and downward propagation amplitude in our histogramequalized flux maps) with the amplitude of the chromospheric three-minute oscillation which constitutes internetwork grains.

In order to emphasize the oscillatory nature of internetwork grains, we define "bright and dark $\mathrm{K}_{2 \mathrm{~V}}$ points" in a special manner. For each internetwork pixel and for each exposure $n=5, \ldots, 74$ in the 78 -image $\mathrm{Ca}_{\text {II }} \mathrm{K}_{2 \mathrm{~V}}$ sequence, the extreme brightness differences are evaluated between the $\mathrm{K}_{2 \mathrm{~V}}$ brightness at exposure time and in the averaged pairs of bracketing exposures with $\Delta n= \pm 2,3,4$. Thus, for each internetwork pixel in image $n$ the three differences $I_{n}-\left(I_{n-\Delta n}+I_{n+\Delta n}\right) / 2$ for 


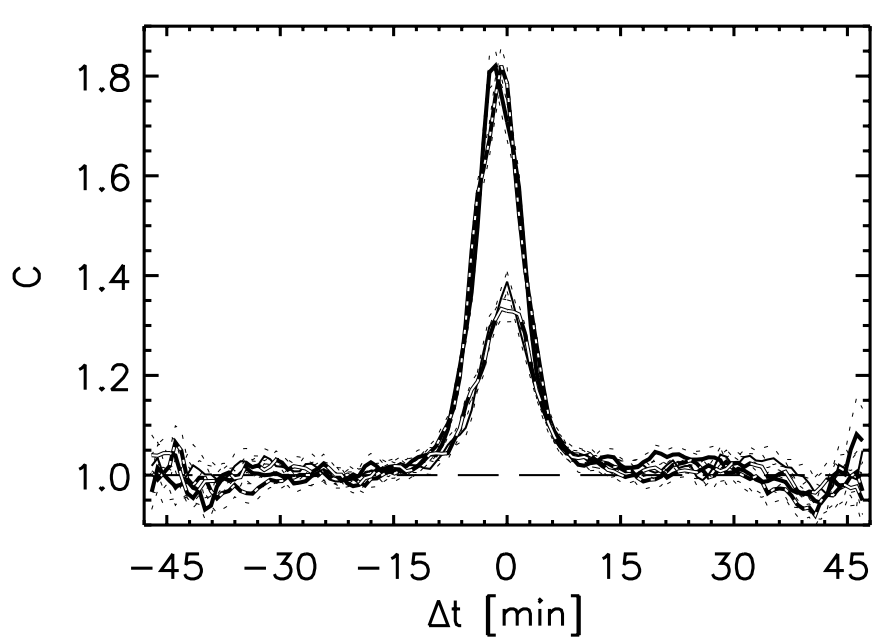

Fig. 10. Time-delay correspondence between acoustic flux in the photosphere and $\mathrm{Ca}_{\mathrm{II}} \mathrm{K}_{2 \mathrm{~V}}$ oscillatory amplitude in the chromosphere, in internetwork areas. There are four curves, a pair for the $5 \%$ bin of pixels with maximum upwards propagating flux together constituting the higher peak and a pair for the 5\% bin of pixels with maximum downward propagating flux together constituting the lower peak. In each pair the solid curve is for the $5 \%$ brightest oscillation pixels ("bright points") and the (nearly overlapping) blocked curve is for the 5\% darkest oscillation pixels ("dark points"). The thin dashed curves specify $1 \sigma$ variances.

$\Delta n=2,3,4$ and $\Delta t / \Delta n=47 \mathrm{~s}$ are determined. The largest of these three differences is denoted the bright point value of that pixel in exposure $n$, the smallest the dark point value. They are a measure of instantaneous $\mathrm{K}_{2 \mathrm{~V}}$ brightness oscillation amplitude which may be compared, again averaging over all pertinent pixels, with the underlying acoustic flux in correspondence analysis.

The motivation to admit a wider band of periodicity than just three minutes $(\Delta n=2)$ is that the chromospheric internetwork oscillation is a fairly wide-band phenomenon, as is indeed illustrated by the temporal excursions of the $\mathrm{K}_{2 \mathrm{~V}}$ brightness in Fig. 4 and the peak recurrency in Fig. 9. Note that the chromospheric "three-minute" oscillation is broad-band even when sampled higher up in the Ca II $\mathrm{K}$ line center and that the $0.6 \AA$ Halle-filter passband used here adds the upper-photospheric wing whiskers into the oscillatory signal (Beckers \& Artzner 1974; cf. Krijger et al. 2001).

Figure 10 shows the result. The correspondence reaches very high values, nearly twice the random-draw likelihood, between extreme upward flux and extreme oscillatory $\mathrm{K}_{2 \mathrm{~V}}$ enhancement. The statistical significance is very high (the $1 \sigma$ variances lie very close to the correspondence curves). A smaller but equally significant correspondence with extreme oscillatory enhancement holds for downward propagating flux maxima (lower peak).

The width of these correspondence peaks is considerably narrower than for the granulation-flux correspondence curves in Fig. 8. This may result from $\mathrm{K}_{2 \mathrm{~V}}$ brightness features with fast apparent motion over the solar surface that are lost in timedelay samplings by moving off the pixel.

At our low time resolution there is no difference in excessive bright-point and dark-point correspondence with excessive flux. This curve equality indicates that the internetwork grain phenomenon is indeed oscillatory, with extreme brightening and darkening occurring in equal amounts as response to forcing from below. However, there is a difference between positive and negative flux, with the upward propagation having markedly larger correspondence.

The two high peaks (upward propagation) show a time delay (excessive bright point after excessive upward flux) of about two minutes which corresponds well with the sound speed travel time over the formation height difference (about $7 \mathrm{~km} \mathrm{~s}^{-1}$ and $800 \mathrm{~km}$, respectively, cf. Carlsson \& Stein 1997). The two low peaks have negligible time delay, which may imply that the pertinent downward propagation events followed on prior wave excitation after a similar delay.

\section{Conclusion}

The time-delay scatter plots in Fig. 6 and the time-delay correspondence plot in Fig. 10 show that extreme acoustic events in the photosphere which generate excessive amounts of upward propagating acoustic waves have larger-than-random likelihood to be accompanied or followed by excessive Ca II $\mathrm{K}_{2 \mathrm{~V}}$ oscillation amplitude in the internetwork chromosphere. The correlation is far from one to one but highly significant.

It would be surprising to find a one-to-one correlation since weaker granular pistoning is likely to produce smaller brightness modulation all over the place, large fractions of the acoustic wave field share in global or at least non-local $p$-mode oscillations with complex interference patterns, atmospheric gravity waves contribute additional brightness modulation, and internetwork flashers and other magnetic fields in the internetwork may play a role as well. Our results suggest that the most excessive acoustic excitation events poke through this sea of motion to produce the fractional correlation with excessive $\mathrm{Ca}$ II $\mathrm{K}_{2 \mathrm{~V}}$ modulation established here.

The time resolution of our data is not good enough to measure piston-response connectivity and time delay with higher precision than done here. New observations with a faster image cadence have been collected at the Dunn Solar Telescope in the meantime. In combination with wavelet analysis they may serve to study acoustic pistoning and wave propagation from photosphere to chromosphere in more detail.

Acknowledgements. N. M. Hoekzema is grateful to the National Solar Observatory/Sacramento Peak and its staff for inviting him to work on these data and for their hospitality. T. R. Rimmele and R. J. Rutten acknowledge invitations to the High Altitude Observatory in Boulder since this analysis was inspired by a colloquium held there by the first and attended by the second. We are indebted to the Dunn Solar Telescope staff for excellent technical support and assistance in the observing, to J. M Krijger for diagnosing a programming error, and to him, D. E. Innes and the referee for improvements to the manuscript.

\section{References}

Beckers, J. M., \& Artzner, G. 1974, Sol. Phys., 37, 309

Brandt, P. N., Rutten, R. J., Shine, R. A., \& Trujillo Bueno, J. 1992, in Cool Stars, Stellar Systems, and the Sun, ed. M. S. Giampapa, \& J. A. Bookbinder (Proc. Seventh Cambridge Workshop), ASP Conf. Ser., 26, 161 
Brandt, P. N., Rutten, R. J., Shine, R. A., \& Trujillo Bueno, J. 1994, in Solar Surface Magnetism, ed. R. J. Rutten, \& C. J. Schrijver, NATO ASI Ser. C 433 (Dordrecht: Kluwer), 251

Carlsson, M., \& Stein, R. F. 1997, ApJ, 481, 500

Cram, L. E., \& Damé, L. 1983, ApJ, 272, 355

Damé, L. 1985, in Theoretical Problems in High Resolution Solar Physics, ed. H. U. Schmidt, MPA/LPARL Workshop (München: Max-Planck-Institut für Physik und Astrophysik MPA 212), 244

Damé, L., Gouttebroze, P., \& Malherbe, J.-M. 1984, A\&A, 130, 331

Damé, L., \& Martić, M. 1987, ApJ, 314, L15

Damé, L., \& Martić, M. 1988, in Advances in Helio- and Asteroseismology, ed. J. Christensen-Dalsgaard, \& S. Frandsen (Dordrecht: Reidel), IAU Symp., 123, 433

Georgobiani, D., Kosovichev, A. G., Nigam, R., Nordlund, Å., \& Stein, R. F. 2000, ApJ, 530, L139

Goode, P. R., Gough, D., \& Kosovichev, A. G. 1992, ApJ, 387, 707

Goode, P. R., Strous, L. H., Rimmele, T. R., \& Stebbins, R. T. 1998, ApJ, 495, L27

Hale, G. E., \& Ellerman, F. 1904, ApJ, 19, 41

Hoekzema, N. M., \& Brandt, P. N. 2000, A\&A, 353, 389

Hoekzema, N. M., Brandt, P. N., \& Rutten, R. J. 1998a, A\&A, 333, 322

Hoekzema, N. M., \& Rutten, R. J. 1998, A\&A, 329, 725

Hoekzema, N. M., Rutten, R. J., Brandt, P. N., \& Shine, R. A. 1998b, A\&A, 329, 276

Hofmann, J., Steffens, S., \& Deubner, F. L. 1996, A\&A, 308, 192

Krijger, J. M., Rutten, R. J., Lites, B. W., et al. 2001, A\&A, 379, 1052

Kumar, P. 1994, ApJ, 428, 827

Kumar, P., \& Basu, S. 1999, ApJ, 519, 396

Lites, B. W., Rutten, R. J., \& Berger, T. E. 1999, ApJ, 517, 1013

Lites, B. W., Rutten, R. J., \& Kalkofen, W. 1993, ApJ, 414, 345

Nigam, R., \& Kosovichev, A. G. 1999a, ApJ, 510, L149

Nigam, R., \& Kosovichev, A. G. 1999b, ApJ, 514, L53

Nindos, A., \& Zirin, H. 1998, Sol. Phys., 179, 253
Oliviero, M., Severino, G., Straus, T., Jefferies, S. M., \& Appourchaux, T. 1999, ApJ, 516, L45

Rast, M. P. 1999, ApJ, 524, 462

Remling, B., Deubner, F. L., \& Steffens, S. 1996, A\&A, 316, 196

Restaino, S. R., Stebbins, R. T., \& Goode, P. R. 1993, ApJ, 408, L57

Rimmele, T. R., Goode, P. R., Harold, E., \& Stebbins, R. T. 1995, ApJ, 444, L119

Rutten, R. J., de Pontieu, B., \& Lites, B. W. 1999, in High Resolution Solar Physics: Theory, Observations, and Techniques, ed. T. R. Rimmele, K. S. Balasubramaniam, \& R. R. Radick, Procs. 19th NSO/Sacramento Peak Summer Workshop, ASP Conf. Ser., 183, 383

Rutten, R. J., \& Uitenbroek, H. 1991, Sol. Phys., 134, 15

Sivaraman, K. R. 1991, in Mechanisms of Chromospheric and Coronal Heating, ed. P. Ulmschneider, E. Priest, \& B. Rosner, Heidelberg Conf. (Berlin: Springer Verlag), 44

Sivaraman, K. R., Gupta, S. S., Livingston, W. C., et al. 2000, A\&A, 363,279

Sivaraman, K. R., \& Livingston, W. C. 1982, Sol. Phys., 80, 227

Skartlien, R. 1998, 3D modeling of solar convection and atmosphere dynamics (Inst. Theor. Astrophys. Oslo: Ph.D. Thesis)

Skartlien, R., \& Rast, M. P. 2000, ApJ, 535, 464

Skartlien, R., Stein, R. F., \& Nordlund, Å. 2000, ApJ, 541, 468

Stebbins, R., \& Goode, P. R. 1987, Sol. Phys., 110, 237

Stebbins, R. T., Goode, P. R., \& Hill, H. A. 1983, Sol. Phys., 82, 163

Steffens, S., Hofmann, J., \& Deubner, F. L. 1996, A\&A, 307, 288

Straus, T., Severino, G., Deubner, F. L., et al. 1999, ApJ, 516, 939

Strous, L. H. 1994, Dynamics in Solar Active Regions: Patterns in Magnetic-Flux Emergence (Utrecht University: Ph.D. Thesis)

Strous, L. H., Goode, P. R., \& Rimmele, T. R. 2000, ApJ, 535, 1000 von Uexküll, M., \& Kneer, F. 1995, A\&A, 294, 252

Wellstein, S., Kneer, F., \& von Uexküll, M. 1998, A\&A, 335, 323

Worden, J., Harvey, J., \& Shine, R. 1999, ApJ, 523, 450 